\title{
Understanding the structural determinants of object confusion in memory: An assessment of psychophysical approaches to estimating visual similarity
}

\author{
GENEVIÈVE DESMARAIS and MIKE J. DIXON \\ University of Waterloo, Waterloo, Ontario, Canada
}

\begin{abstract}
Computer-generated shapes varying on visual dimensions such as curvature, tapering, and thickness have been used to investigate identification deficits in the category-specific visual agnosia (CSVA) Patient E.L.M.. However, whether the implemented variations on each of these dimensions were perceived by novice observers as "similar amounts of change" is unknown. To estimate distance in psychophysical shape space, sets of shapes were developed using two different scaling methods—an objective method based on visual search, and a subjective method based on judgments of similarity-and a third approach that did not involve scaling. How well each method estimated psychophysical shape space was assessed by measuring the confusions within memory among the shapes. The results suggested that, although neither of the approaches perfectly reflected psychophysical shape space, subjective scaling was a better estimator of distance in psychophysical shape space than were other approaches. The number of confusions produced on each set of shapes was used to develop a new set of shapes that accurately estimated distance in psychophysical shape space. These results suggest that a combination of approaches is preferable in order to accurately estimate distance in psychophysical shape space.
\end{abstract}

What are the main cognitive principles underlying the identification of objects? A clue to these principles comes from the phenomenon of category-specific visual agnosia (CSVA), a condition in which identification deficits following temporal lobe damage selectively target specific classes of objects, but not others. Typically items such as animals, birds, fruits, and vegetables are impaired following brain damage, whereas items from categories such as furniture, tools, and clothing are spared. Investigating the cognitive mechanisms that would allow a CSVA patient to be able to identify tools, but not large felines (e.g., lion, tiger, leopard) could ultimately allow us to uncover the principles humans use to store objects in memory as well as to compare perceived objects to stored objects for the purpose of identification.

\section{Visual Similarity}

A number of researchers have proposed that category differences observed in typical CSVA patients arise because of differences in the structural similarity between

This research was supported by Natural Sciences and Engineering Research Council Grant PIN 203130-98 awarded to the second author. The authors offer a special thanks to Sarah Ann Greatrix, Sarah Shaughnessy, Sara Kim, and Ruth Grandon for their help carrying out these studies and to Daniel Smilek for some helpful discussions on visual search. Correspondence concerning this article should be addressed to G. Desmarais, Psychology Department, University of Waterloo, 200 University Avenue West, Waterloo, ON, N2L 3G1 Canada (e-mail: gdesmara@ watarts.uwaterloo.ca). the items comprising certain categories. Forde, Francis, Riddoch, Rumiati, and Humphreys (1997), as well as Tranel, Logan, Frank, and Damasio (1997), observed poorer naming performance for line drawings of objects comprising categories of structurally similar exemplars. These authors reasoned that patients who are impaired at identifying a lion, a tiger, and a leopard, but are unimpaired at identifying a hammer, a saw, and a screwdriver, show this pattern at least in part, because the cats are more visually similar to each other than the tools are. In order to quantify object similarity, two main approaches have been used: subjective similarity ratings and objective measures of object overlap. Objective measures use various algorithms to measure the degree of structural overlap between depictions of objects; subjective measures use similarity ratings between two or more objects.

Tranel et al. (1997) used within-category shape overlap as an objective measure of within-category shape similarity. They defined a region of interest (ROI), which was essentially the outer contour of each object used. These ROIs were aligned according to their longest naturally occurring axis, and the amount of overlap between the ROIs from different objects was compared. This approach yielded a similarity measure based on the amount of overlap between the shapes of objects comprising a given category. The authors reported that the categories that are typically impaired in CSVA patients had the highest amount of shape similarity, and the categories that are typically spared in CSVA patients had the least amount of shape similarity. Their findings indicate that greater similarity 
leads to greater identification impairments in patients with temporal lobe damage.

With line drawings, even though we can get at an estimation of object similarity using measures such as contour overlap, the underlying shape primitives of the forms depicted in line drawings are still unspecified (Dixon, Bub, \& Arguin, 1997). That is, although contour overlap may provide an empirical measure of shape similarity, line drawings contain a myriad of complex features, and there is currently no way of assessing the degree to which patients actually use object contours to identify objects, rather than other types of information available in the line drawings. As an example, a pear and a light bulb have a higher degree of contour overlap than a pear and an apple, but CSVA patients would never confuse pears with light bulbs, although they might confuse apples and pears. Here the point is not that other factors must be involved in visual object identification (the presence of stems, semantic similarity etc.), but rather that one cannot specify a priori which of these factors are preferentially used to visually identify line drawings. Accordingly, because we cannot specify which aspects of line drawings allow us to distinguish between two similar items, shape similarity as determined by shape overlap is at best an ambiguous measure of within-category similarity that may or may not bear a causal relation in determining how patients with CSVA, or healthy observers, identify objects.

\section{Novel Shapes}

One way to circumvent the pitfalls of line drawings is to use simple, novel, and empirically specifiable computergenerated shapes instead of line drawings of objects. ${ }^{1}$ Starting with one ellipse-shaped "origin shape" (see shape $0,0,0$ in Figure 1) it is possible to transform its appearance along any of three dimensions: curvature, tapering, and thickness. Note that the elliptical origin shape can be transformed along single or multiple dimensions (e.g., along just curvature, along curvature and tapering, or along curvature, tapering, and thickness, etc.). Because the degree of transformation of the shapes can be precisely specified, it is possible to maintain empirical control over the visual similarity of the shapes comprising a shape set. These shapes can then be plotted in three-dimensional space according to the amount of transformation given to them on each dimension.

Previously, to create sets of shapes like these, researchers have used intuitively similar amounts of transformation on each dimension (Dixon et al., 1997; Dixon, Bub, Chertkow, \& Arguin, 1999; Dixon, Desmarais, Gojmerac, Schweizer, \& Bub, 2002). After creating sets of shapes in which the exemplars differed from one another in known, empirically specifiable ways, Dixon et al. (1997) then used these shapes to investigate the influence of shape similarity in object identification performance in a patient with category-specific visual agnosia. Recall that with line drawings, in addition to quantifying similarity using objective measures such as contour overlap, researchers have also used subjective measures of shape similarity.

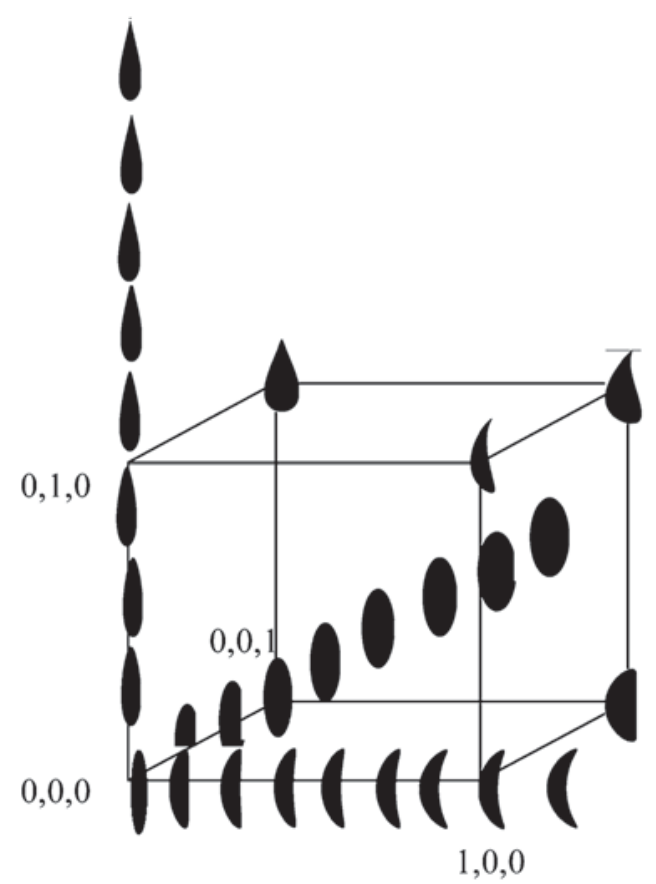

Figure 1. Three-dimensional psychophysical shape space. The coordinates refer to values of curvature $(1,0,0)$, tapering $(0,1,0)$, and thickness $(0,0,1)$.

Dixon et al. (1997) showed that participants' subjective ratings of shape similarity for these computer-generated shapes were not a good predictor of how easily their CSVA Patient E.L.M. could identify these shapes. In the Dixon et al. study, two types of shape sets were contrasted. One set consisted of four exemplars that differed from one another on the single dimension of thickness. In the other set, each exemplar had a unique combination of thickness and curvature (Shape $1=$ thin and uncurved, Shape $2=$ thin and curved, Shape $3=$ thick and uncurved, Shape $4=$ thick and curved). E.L.M. was better at identifying the shapes whose members varied on the single dimension of thickness than the shapes composed of different combinations of thickness and curvature (see Experiment 1, Dixon et al., 1997). This finding did not align with healthy participants' subjective ratings of visual similarity. They rated the shapes varying on the single dimension of thickness as being more similar to one another than the shapes bearing different combinations of thickness and curvature.

In sum, although different means of assessing shape similarity have proven beneficial in accounting for object identification performance, they also have their limitations; objective measures of contour overlap leave unspecified the degree to which patients actually use this information in identifying objects (the pear-light bulb problem), and subjective measures of similarity do not always account for patients' identification performance.

E.L.M.'s pattern of identification problems with the simple computer-generated shapes caused Dixon et al. (1997) to refine their definition of shape similarity. Dixon et al. 
(1997) proposed that E.L.M.'s deficit in shape identification occurred when "identification of a given shape involves extracting from memory specific values along more than one relevant shape dimension" (pp. 10991100). Thus the type of shape similarity that poses problems for CSVA patients like E.L.M. is quite specific. If shapes are similar in a unidimensional shape space, patients can compensate for their deficits by focusing all of their attention on one dimension and can identify these shapes quite well. By contrast, when shapes within a set bear different combinations of multiple shape dimensions, severe identification deficits ensue. Therefore, in order to develop an understanding of shape similarity, and thus of shape identification, Dixon et al. (1997) showed that it is necessary to consider the shapes' relations in a multidimensional shape space.

\section{Psychophysical Shape Spaces}

An example of a three-dimensional shape space is presented in Figure 2. The relevant axes in this space are curvature, tapering, and thickness. The position of a given shape reflects the degree or amount of each feature present in that shape (see Figure 2). Therefore, the position of a given shape in three-dimensional shape space can be described as a set of Cartesian coordinates that indicate the amount of a particular feature (curvature, tapering, or thickness) possessed by that shape. For example, the shape in position $0,0,1$ in Figure 2 has no curvature or tapering but is thick. (For simplicity of coding, we have coded the thinnest shapes in the set as having 0 thickness. These can be thought of as having the minimal thickness to be considered shapes rather than lines.)

In this shape space, some pairs of shapes lie close together and some lie further apart. The shapes labeled 0,0,0 and $0,0,1$ are closer to each other than are the shapes labeled
$0,0,0$ and 1,1,1, for example. The distance between two shapes can be measured using city-block (CB) distancethe sum of the differences between shapes on each of the dimensions (Shepard, 1987). Thus shapes labeled 0,0,0 and 0,0,1 lie one CB apart, shapes labeled 0,0,0 and 1,0,1 lie two CBs apart and 0,0,0 and 1,1,1 lie three CBs apart.

Shapes that are closer together in shape space have fewer distinguishing features than shapes that are further apart. Shapes $0,0,0$ and 0,0,1 share the same value on two dimensions (curvature and tapering), but differ on thickness. Thus, for this pair, thickness is the distinguishing feature. Shape pairs differ in terms of their number of distinguishing features. Shapes 0,0,0 and 0,0,1 have one distinguishing feature, whereas shapes $0,0,0$ and 1,0,1 have two distinguishing features, and shapes $0,0,0$ and $1,1,1$ have three distinguishing features. Note that the number of distinguishing features equals the number of $\mathrm{CBs}$ that separate two shapes. Shapes that are closer to each other in psychophysical shape space have fewer distinguishing features and are therefore more similar than shapes that are further apart.

The similarity between items is correlated with speed and error performance on sorting tasks. Lockhead and King (1977) reported that "stimuli closely adjacent in similarity space are more difficult to process and take longer to sort whereas stimuli more distant in psychological space are easily distinguishable and are sorted quickly" (p. 440). Therefore, in a shape identification task, confusions should occur mostly between shapes adjacent in shape space and less often between shapes that are further apart. Thus, when people are asked to identify a novel shape or the drawing of an object, if they make errors, the errors should be between items that are close together in shape space because these objects have fewer distinguishing features than objects that are further apart. Therefore,

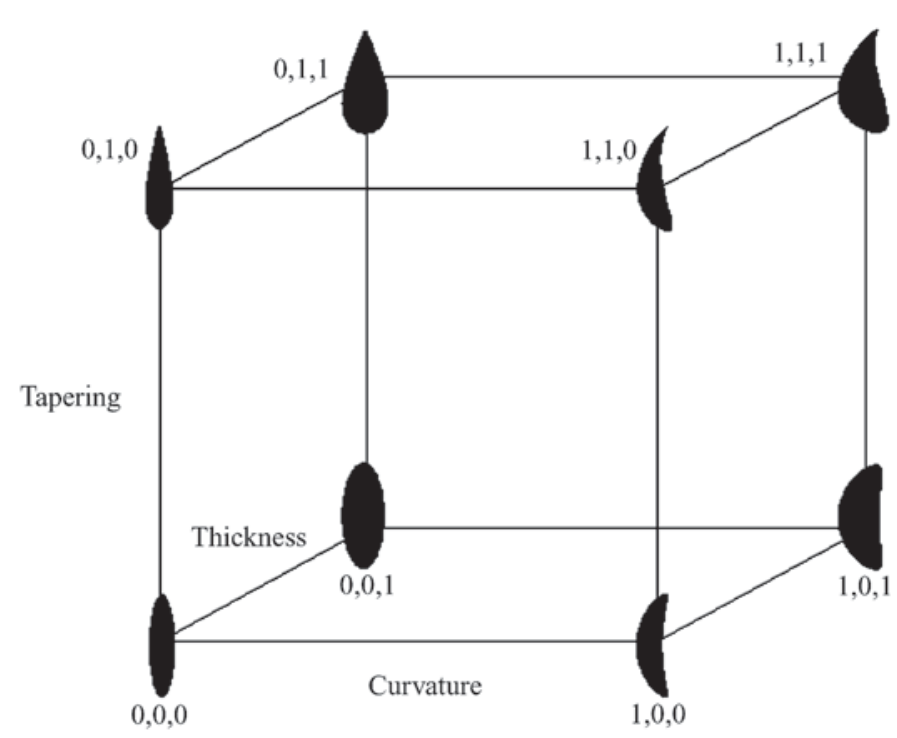

Figure 2. Three-dimensional psychophysical shape space. The coordinates refer to values of curvature $(1,0,0)$, tapering $(0,1,0)$, and thickness $(0,0,1)$. 
if shape sets are defined by multiple visual dimensions, shapes that lie on adjacent points in shape space should be confused the most often whereas shapes that are separated by the greatest distance in shape space should be confused the least often.

In the shape sets used by Dixon et al. (1997), the assumption was that the intuitively derived perturbations of the origin shape along each axis were roughly equivalent. The researchers further presumed that all shapes that lie equidistant to one another would bear comparable degrees of similarity to each other and hence be confused equally often. Concretely, shapes 0,0,0 and 0,0,1 would bear the same resemblance and thus be confused equally often as shapes $0,0,0$ and $1,0,0$ would.

One means of assessing the similarity of the shapes used by Dixon et al. (1997) is to compare the number of confusions that patients make when trying to identify these shapes. Dixon et al. (2002) presented the CSVA Patient E.L.M. with shapes varying on thickness and curvature (see the top face of the cube shown in Figure 2). On learning trials, the four shapes were paired with the names of four stringed musical instruments: guitar, banjo, violin, and cello. On test trials, shapes were presented alone, and E.L.M. had to generate the label with which it was associated on learning trials. Generally, E.L.M. confused shapes that were adjacent (one CB apart) in this two-dimensional shape space more often than shapes that were on diagonally opposed corners of this shape space (two CBs apart). However, the four pairs that were one $\mathrm{CB}$ apart did not generate identical numbers of confusions; some pairs generated a greater number of confusions than did other pairs. For example, E.L.M. produced 32 confusions between shapes 1,1,0 and 1,1,1 (differing in thickness), but only 12 confusions between shapes $0,1,1$ and 1,1,1 (differing in curvature), even though both pairs lay one $\mathrm{CB}$ apart.

Although it is possible that differences in these confusion rates may reflect the semantic proximity of the labels (i.e., a guitar may be closer to a banjo than to a violin), it is also possible that the shape pairs that generated a greater number of confusions were actually more psychologically similar in shape for E.L.M. than the pairs that generated fewer confusions. This may arise because the distinguishing feature of curvature was much more salient for E.L.M. than the distinguishing feature of thickness. Thus the pair of shapes that differed on curvature was confused less often than the pair of shapes that differed on thickness. Even though the two shapes were intuitively equidistant from the origin shape for the experimenters who created these shapes, for E.L.M., one shape could have been subjectively perceived as more different from the origin shape than was the other shape.

Although it is possible that E.L.M.'s brain damage may have made certain object features more salient than others, it is equally possible that the acquired expertise of the experimenters who spent hours creating these shapes may have altered how intuitively similar various pairs of shapes appeared and distorted the shape space used to test
E.L.M.. In other words, what were equidistant pairs of shapes for the experimenter might have been very discrepant pairs of shapes to a novice (i.e., one pair being much closer in psychological distance than the other pair).

Clearly, what is needed is a means of capturing the psychological distance between shapes for novice observers. The ultimate goal of the current research was to create a psychophysically scaled shape space that would be useful for testing patients with object identification problems.

\section{Psychophysical Approaches}

Three shape spaces were derived using three different methods. The "control" shape space made no attempt to psychophysically scale the similarities between shape pairs, but simply relied on the intuitions of the experimenters. The purpose of this space was to provide a baseline for evaluating two other methods of psychophysically scaling shapes. We used both an objective and a subjective method of quantifying shape similarity. The "objective" shape space was derived using visual search efficiency. Reasoning that target-distractor similarity influences visual search efficiency, we had various shape pairs serve as targets and as distractors in a search task. Crucial pairs of shapes were then selected using search slopes and intercepts to form this objectively scaled shape space. Finally the "subjective" shape space was derived by having participants estimate the subjective distance between various shape pairs. We selected shape pairs that had equivalent subjective distances to form a subjectively scaled shape space.

Ultimately, we sought to account for object confusions in memory. We reasoned that memory errors should be governed by psychological shape similarity, hence objects that are close together in a psychophysical shape space should be more easily confused than objects that are further apart in psychophysical shape space. Our goal in Experiment 1 was to evaluate whether objective or subjective methods of scaling would better reflect memory confusions.

\section{EXPERIMENT 1}

The shapes that composed all three shape spaces were based on perturbations of a single "origin" cucumber-like shape (see Figure 1). This origin shape was perturbed on the dimensions of curvature, tapering, and thickness using software developed by Greg Dudek (Dudek, Arguin, Dixon, \& Bub, 1997). For example, curvature was minimally increased until it was judged by the experimenter to be just noticeably more curved than the origin shape. This new shape was labeled CUR1. The shape CUR1 was then curved until it was judged by the experimenter to be just noticeably more curved than its former self. This continued until eight curved shapes were generated. The same approach was used to generate eight shapes that varied on tapering and eight shapes that varied in thickness, resulting in a pool of 24 possible perturbations of the origin shape (see Table 1). The control, objective, and subjective 


\begin{tabular}{|c|c|c|c|c|c|}
\hline \multicolumn{6}{|c|}{$\begin{array}{c}\text { Table 1 } \\
\text { Shapes Used in Experiment } 1 \\
\end{array}$} \\
\hline $\begin{array}{l}\text { Shape } \\
\text { Name }\end{array}$ & Shape & $\begin{array}{l}\text { Shape } \\
\text { Name }\end{array}$ & Shape & $\begin{array}{l}\text { Shape } \\
\text { Name }\end{array}$ & Shape \\
\hline THK1 & & TAP1 & & CUR1 & \\
\hline THK2 & & TAP2 & & CUR2 & \\
\hline THK3 & & TAP3 & & CUR3 & \\
\hline THK4 & & TAP4 & & CUR4 & \\
\hline THK5 & & TAP5 & & CUR5 & \\
\hline THK6 & & TAP6 & & CUR6 & \\
\hline THK7 & & TAP7 & & CUR7 & \\
\hline THK8 & & TAP8 & & CUR8 & \\
\hline
\end{tabular}

Note-THK, thick; TAP, tapered; CUR, curved. All shapes were $81 \mathrm{~mm}$ in length and were presented at $7.69^{\circ}$ of visual angle.

spaces each contained a quadruplet of shapes: the origin shape plus three of these perturbations (one curved, one tapered, and one thick shape).

\section{Control Shape Space}

The control space comprised the origin shape plus the CUR1, TAP1, and THK1 shapes selected from the pool of 24 generated shapes. These latter shapes contained the most minimal perturbations of the origin shape on each of the three respective dimensions. In this control space, no attempt was made to scale the psychological similarity of the shapes within the space. The remaining two shape spaces were each derived using objective and subjective scaling. The rationale for how these spaces were derived, and the resulting shapes that were selected to form the objective and subjective shape spaces, are presented below.

\section{Objective Shape Space}

A number of studies (e.g., Tomogana \& Matsuzawa, 1992) have used indirect measures such as voice response times (RTs) to index the similarities among shapes or choice-RT to index interobject similarity between letters of the alphabet (Podgorny \& Garner, 1979). In a visual search task, participants look for a predetermined target embedded in a display of distractors. In visual search with shapes, when distractor shapes were presented at nonuniform orientations to prevent pop-out, Dixon et al. (1998) showed that two factors influenced target-detection search times: the number of distractors in the display and the target-distractor similarity. The closer the target was to distractors in shape space, the longer the search RTs. To develop our objectively scaled shape space, we varied both the number of distractors in a visual search display and the target-distractor similarity. Participants' RTs for detecting a target shape were recorded, and we then used these RTs to select three shapes (one curved, one tapered, and one thick) that were equal distances away from the origin shape.

Sixty-five undergraduate students from the University of Waterloo were presented with displays of two, four, or eight shapes. The target was always the origin shape, ${ }^{2}$ and the distractors were one of the 24 shapes in the shape pool (multiple copies of the same shape were used as the distractors within a given display size). Participants were presented with displays containing two, four, or eight shapes, and their task was to indicate via a keypress whether the origin shape was present or absent in the display (the present:absent ratio was 4:1). Shapes appeared in one of eight locations on the screen at $12.24^{\circ}$ of visual angle away from fixation (see the eight-shape display in Figure 3), and in one of eight orientations: $0^{\circ}, 45^{\circ}, 90^{\circ}, 135^{\circ}, 180^{\circ}$, $225^{\circ}, 270^{\circ}$ and $315^{\circ}$, and both targets and distractors appeared equally often in each position and each orientation. During each trial, participants were first presented with a fixation point in the middle of the screen for $500 \mathrm{msec}$, followed immediately by a display that filled the screen and remained until a response was produced. When the response was made, a 500-msec intertrial interval elapsed before a new trial began. The sequence of events is depicted in Figure 3. Participants were presented with 16 blocks of 60 trials, and the target shape was presented at the beginning of each block.

Mean RTs were calculated for correct responses to target-present trials, for displays containing two, four, and eight shapes. According to Lockhead and King (1977), finding a target (origin shape) embedded within stimuli that are similar to it (i.e., THK1) should produce longer reaction times than finding the same target embedded within dissimilar stimuli (i.e., THK8). Because all the distractors within a given display were the same shape, but different distractors were used in different displays, it was possible to identify RTs associated with each distractor shape (e.g., the RTs associated with the CUR1, CUR2 through CUR8 distractor shapes; the TAP1, TAP2 through TAP8; and the THK1, THK2, through THK8 distractor shapes). Arguably, shapes that possess the same amount of similarity to the target shape are equidistant from the target shape in psychophysical shape space, and therefore should produce similar RTs for the same display size. Thus one should be able to choose one curved, one tapered, and one thick shape that produce similar patterns of RTs across the different set sizes. Sets of slopes and intercepts can be obtained by plotting the RTs for given shapes at each display size (see Figures 4A-4C). Inspecting slopes or intercepts by themselves would not be informative. Three shapes can have very similar intercepts and yet have diverging slopes. Alternatively, three shapes can have similar slopes and yet have widely discrepant intercepts. Shapes that are equidistant in psychophysical shape space should produce both similar intercepts and similar slopes on a reaction-time task. ${ }^{3}$

To identify which three shapes produced the most similar set of slopes and intercepts, 512 possible triads of shapes were formed using one curved shape, one tapered 


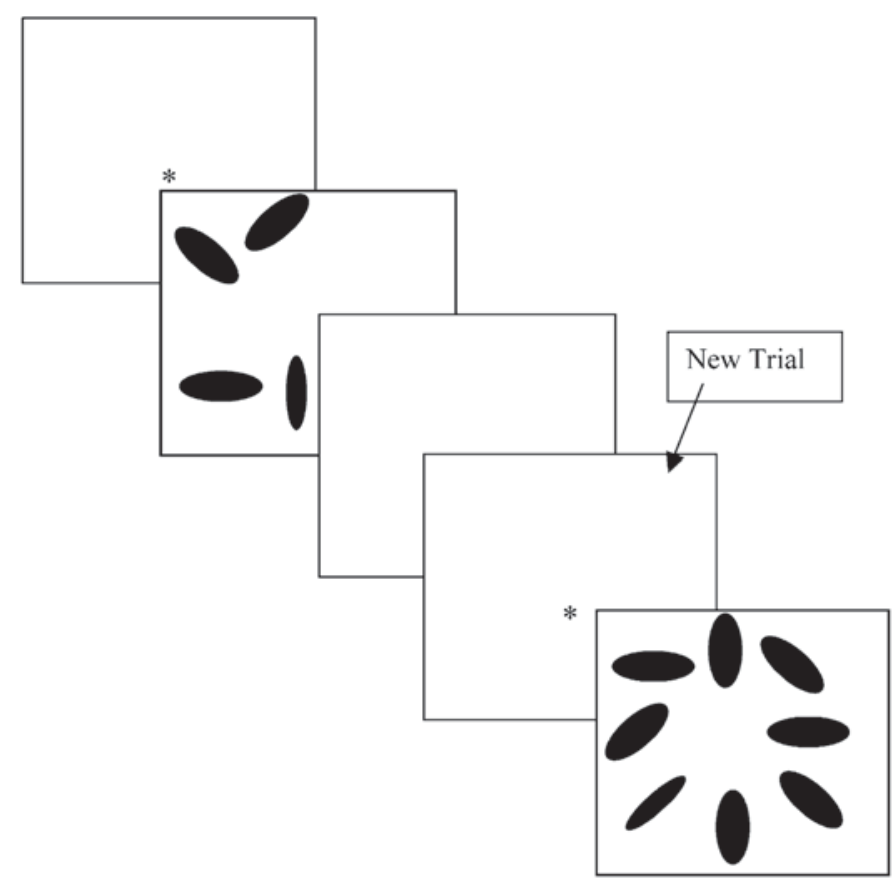

Figure 3. Sequence of events used to scale shapes using a search task.

shape, and one thick shape. For each triad, the standard deviations of the three slopes and the three intercepts were calculated. The 512 triads were then rank-ordered in terms of standard deviations of slopes from least to most, and in terms of standard deviations for intercept from least to most. The ranks for slopes and intercepts of each triad then were summed. The triad of shapes that produced the most similar (least variable) set of slopes and intercepts was the triad of shapes with the lowest rank-sum: CUR7, TAP7, and THK1. This CUR7-TAP7-THK1 triad of shapes constituted our best estimate of shapes that were psychophysically equidistant from the origin shape when

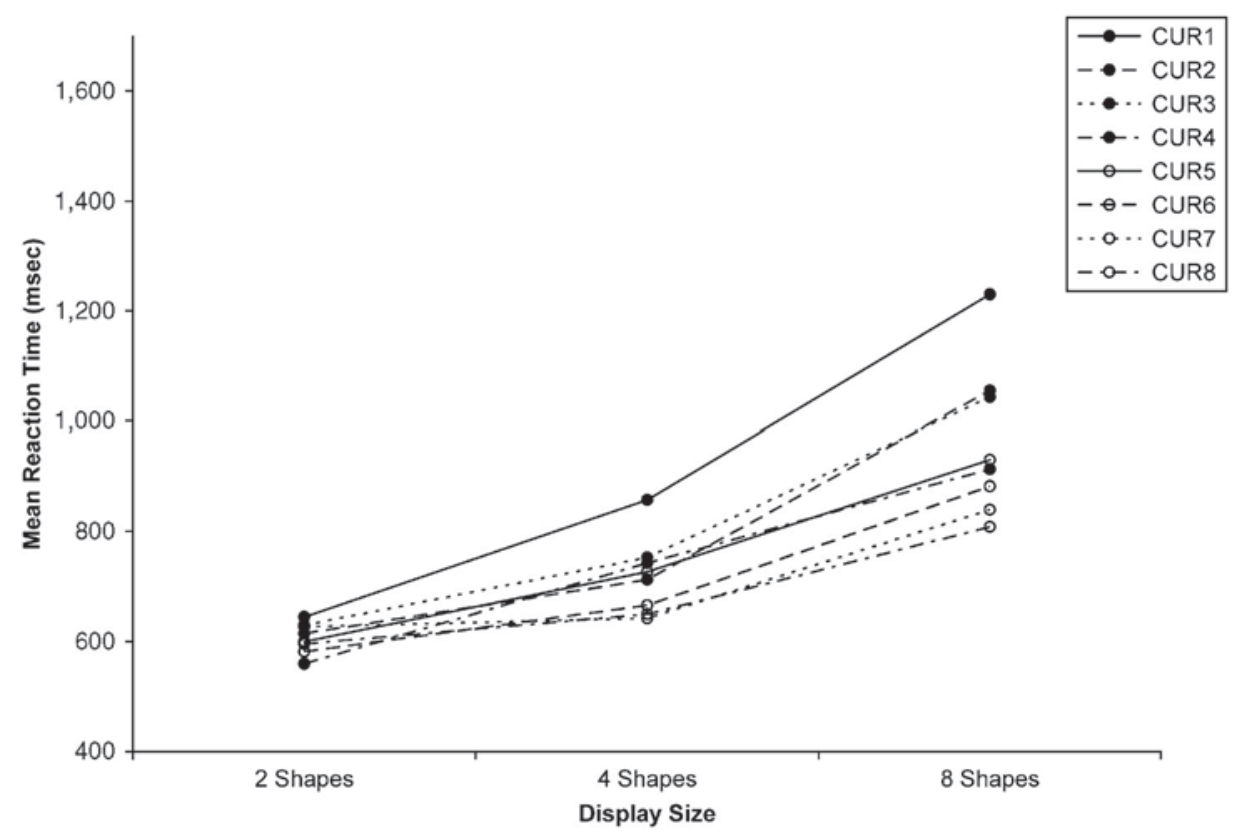

Figure 4A. Mean reaction time (in milliseconds) for displays containing two, four, and eight curved shapes. 


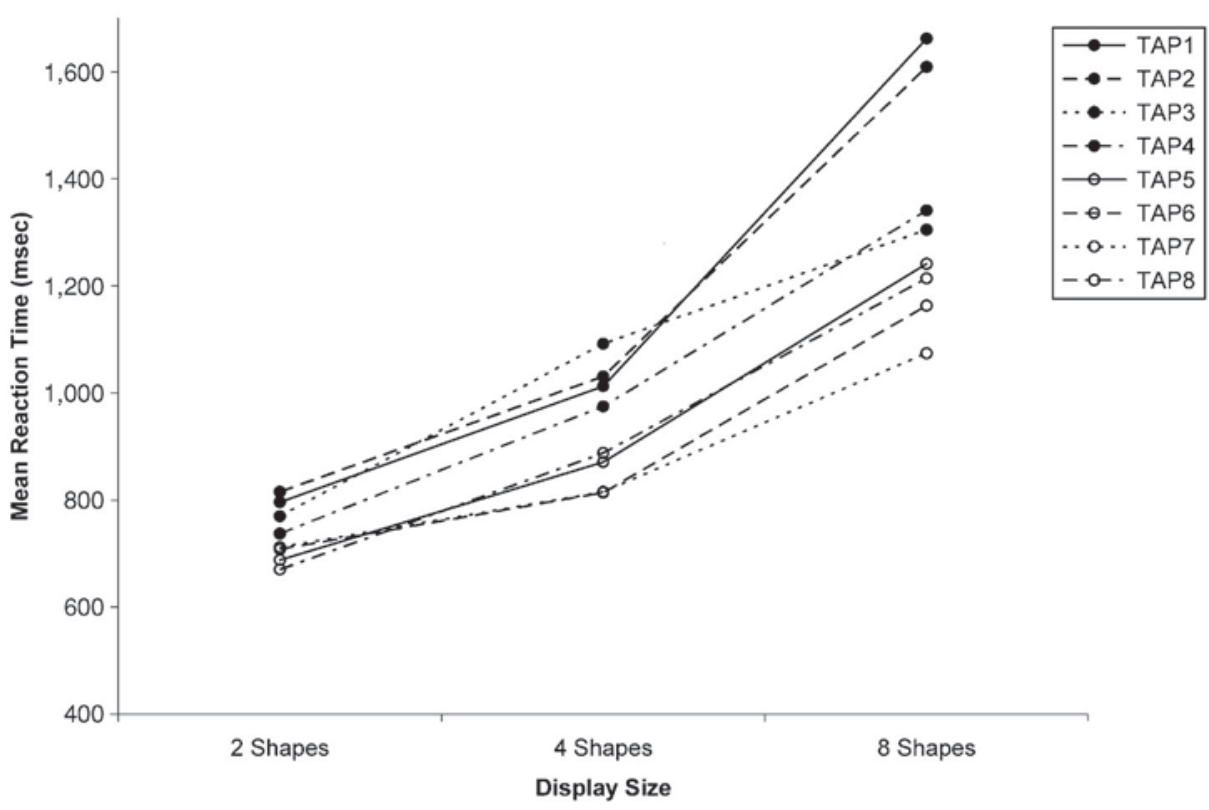

Figure 4B. Mean reaction time (in milliseconds) for displays containing two, four, and eight tapered shapes.

this psychophysical shape space was objectively scaled using visual search.

\section{Subjective Shape Space}

One possible subjective scaling approach would be to present participants with pairs of shapes and to ask them to rate the similarity of these shapes on a seven-point Likert scale. However, when using such similarity ratings, par- ticipants will often focus on the most salient dimensions in making their judgments. Ashby and Lee (1991) reported that participants allocated more attention to the "size" dimension than to the "orientation" dimension in a similarity task, while allotting equal amounts of attention to these two dimensions in an identification task. This diminished the correspondence between similarity judgments of objects and identification of these same objects.

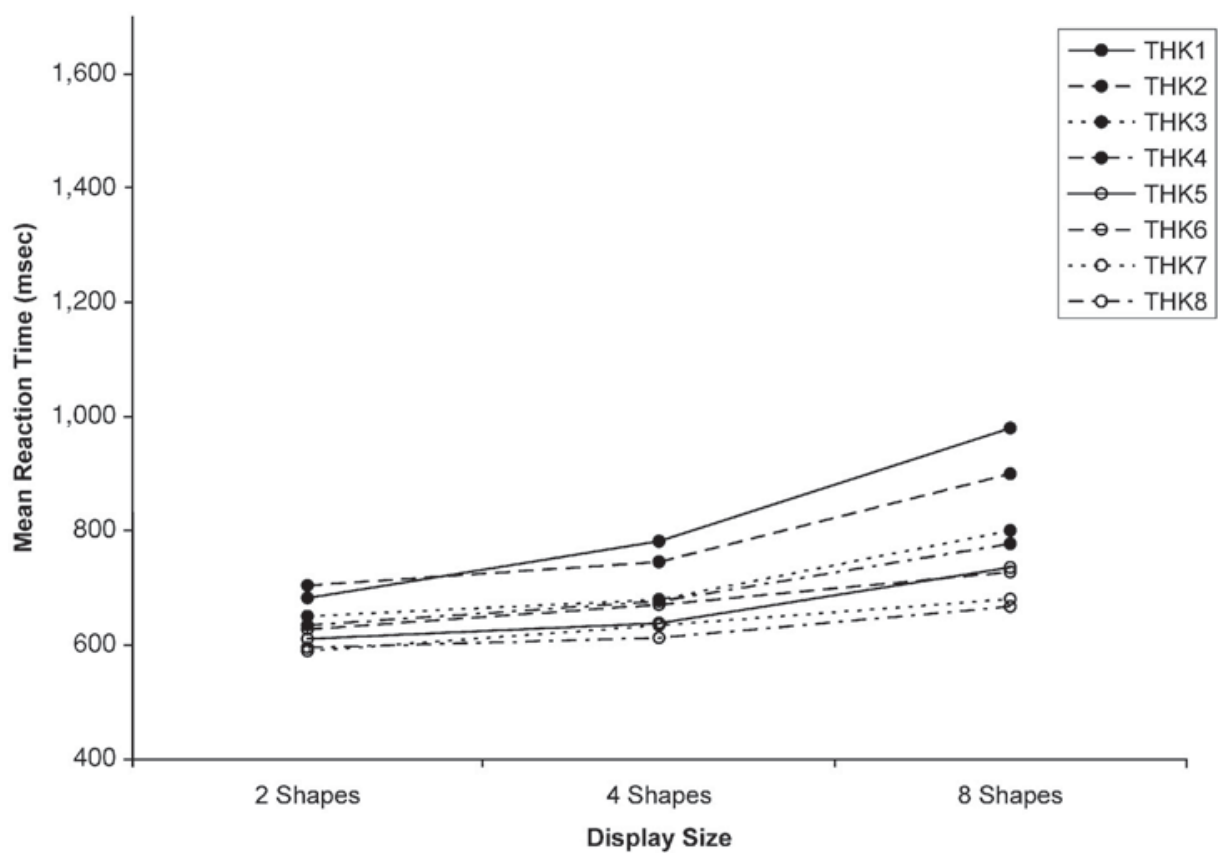

Figure 4C. Mean reaction time (in milliseconds) for displays containing two, four, and eight thick shapes. 
One means of biasing participants to focus on all relevant dimensions within a shape set is to provide a context for similarity estimates. Such contextual anchors were used by Helm (1964) in the color domain (albeit for the purpose of obtaining ratio similarity estimates of colors). Helm presented pairs of anchor colors at fixed points on a grid and asked participants to place a third color at a location on the grid that reflected their perception of the similarity of the target color to the two anchors. This anchor-based strategy was used to generate the subjective space.

Fifty-six undergraduate students from the University of Waterloo were presented with displays containing four constant elements (see Figure 5): two axes forming a large $\mathrm{L}$, a small black dot placed in the middle of the vertical axis of the $\mathrm{L}$, the origin shape placed on the joint of the large L, and the tapered shape identified in the search set placed in the middle of the horizontal axis of the L. ${ }^{4} \mathrm{~A}$ target shape, which varied between trials along a single dimension, was placed in the middle of the screen.

Participants were instructed to evaluate the similarity between the origin shape in the corner of the $\mathrm{L}$ and the tapered shape on the horizontal axis of the $\mathrm{L}$ and to compare this similarity to the similarity between the origin shape and a target shape presented in the middle of the screen. Participants were instructed to place the mouse cursor on the vertical axis of the $\mathrm{L}$, at a position that reflected the similarity between the origin shape and the target shape (the dot in the middle of the vertical axis reflected how similar the origin shape was to the tapered anchor shape). Thus, if the target shape was much more similar to the origin shape than the tapered shape was to the origin shape, participants should click the cursor well below the dot. If the target shape was nearly as similar to the origin shape as the tapered shape was to the origin shape, then they should click the cursor close to the dot. If the target shape was much less similar to the origin shape than the tapered shape was to the origin shape, they should click the cursor at a location well above the dot. Displays remained on the screen until participants produced a response. Only the

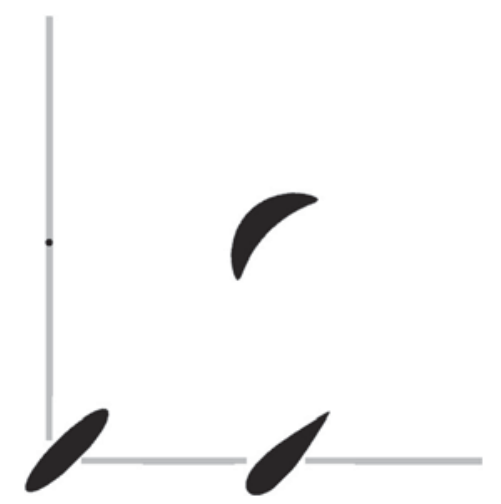

Figure 5. Display used for the subjective scaling of shapes. The two axes were the same length and displayed in green. The black dot was in the center of the ordinate, the teardrop shape was in the center of the absicca. target shape varied between trials, and any given participant was presented with target shapes that varied along a single dimension.

Participants were presented with 20 blocks of nine trials. Within each block, there were nine separate target shapes requiring similarity estimates. One target shape was the tapered anchor shape. Presenting this shape as a target provided a baseline measure for the identification of two other shapes, one curved and one thick, equidistant from the origin shape. The other eight shapes were perturbations of the origin shape along the dimension of curvature (CUR1 to CUR8) or thickness (THK1 to THK8).

The "clicked" position of each shape on the vertical axis was measured in pixels and the mean position for each shape is given in Table 2. The pixel-value data can be construed as "similarity values." At $1,024 \times 768$ resolution, pixel values begin at the top of the screen with 1 and increase down the screen to 768 , therefore a low number indicates low similarity whereas a high number indicates high similarity. The mean position for the anchor indicates participants' similarity estimates when the anchor shape was the target.

To derive which curved shape and which thick shape were as far from the origin shape as the TAP7 shape (i.e., the anchor shape), absolute difference scores between candidate shapes and the TAP7 shape were calculated. When the anchor shape was the target, its mean similarity value placement was 385 (the location 1 pixel below the dot on the vertical arm of the L shape). The thick shape with the value closest to 385 was the THK6 shape (bearing a value of 404). The curved shape with the value closest to 385 was the CUR4 shape (bearing a value of 385). Thus, these shapes were judged to be as similar to the origin shape as the tapered anchor shape.

Figure 6 shows three shape spaces: a control shape space, an objective shape space, and a subjective shape space. In each shape space, there are three shape pairs that lie one $\mathrm{CB}$ apart (origin-curved, origin-tapered, and origin-thick) and three pairs that lie two CBs apart (thicktapered, thick-curved, and tapered-curved). In these psychophysical shape spaces, shapes that are closer together (i.e., one $\mathrm{CB}$ ) should be confused more often than pairs of shapes that are further apart (i.e., two CBs). Thus, one means of evaluating the utility of each scaling method is to see whether shapes that are putatively more similar to one another are confused more often than shapes that are more distinct. Specifically, we sought to assess whether all shape pairs lying one $\mathrm{CB}$ apart yield more confusions in a memory task than shape pairs lying two CBs apart.

Another way of evaluating these scaling methods is to assess whether shapes that are putatively the same distance apart are equally confusable. In each of these shape spaces, we assume that the curved, tapered, and thick shapes are psychophysically equidistant (i.e., one CB) from the origin shape. In a memory task, all pairs of shapes that are one CB from each other should be confused the same number of times. Any deficiencies in the scaling procedure should lead to erroneous shapes being selected. If less-than-ideal shapes are selected, then some pairs of shapes (e.g., the 
Table 2

Mean Position (in Number of Pixels) for

Each Shape in the Direct Scaling Task

\begin{tabular}{cclc}
\hline Shape & Position & Shape & Position \\
\hline THK 1 & 523.79 & CUR 1 & 509.49 \\
THK 2 & 500.89 & CUR 2 & 440.98 \\
THK 3 & 466.07 & CUR 3 & 420.94 \\
THK 4 & 444.68 & CUR 4 & $\mathbf{3 8 5 . 2 8}$ \\
THK 5 & 409.68 & CUR 5 & 360.78 \\
THK 6 & $\mathbf{4 0 4 . 0 4}$ & CUR 6 & 313.28 \\
THK 7 & 353.47 & CUR 7 & 269.25 \\
THK 8 & 341.71 & CUR 8 & 233.35 \\
ANCHOR & 385.89 & ANCHOR & 385.06 \\
\hline
\end{tabular}

Note-THK, thick; CUR, curved. The values in boldface type identify the two shapes equidistant to the origin shape in psychophysical shape space.

origin-thick pair) will be closer in psychophysical shape space than other pairs (e.g., the origin-curved pair). Using similar reasoning, all shape pairs lying two CBs apart should yield similar numbers of confusions in a memory task, and any deficiencies in the scaling procedure being evaluated will lead to violations of this rule.
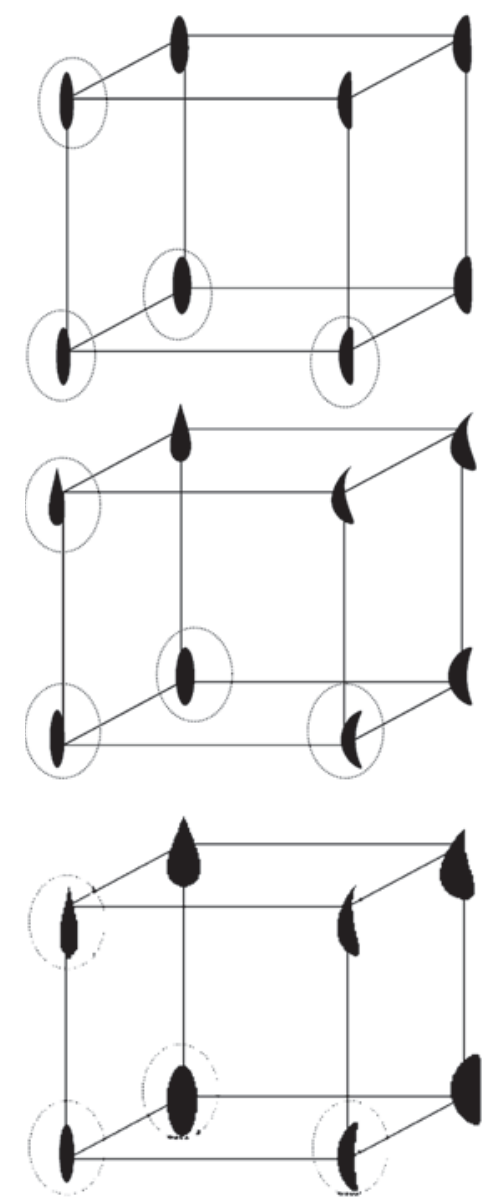

Figure 6. The sets of four shapes derived from the smallest difference (top panel), from the search task (middle panel), and from direct scaling (lower panel).
In sum, we used two methods to evaluate the different scaling methods: (1) seeing if shapes lying one city block apart were confused more often than shapes lying two city blocks apart when confusions were measured in the context of a memory task and (2) seeing if all shapes lying one city block apart were confused equally often and if all shapes lying two city blocks apart were confused equally often in this same memory task.

To evaluate each shape space, we assessed confusions between shapes in the following shape identification task.

\section{Method}

\section{Participants}

Participants were 118 undergraduate students registered in the Introduction to Psychology course at the University of Waterloo; each received one credit for their participation in the experiment.

\section{Materials}

Three sets of eight shapes were constructed. These three shape sets are shown in Figure 6. The first set was based on the unscaled quadruplets of shapes (control set: top cube in Figure 6); the second set was based on the quadruplet of shapes scaled by visual search (objective set: middle cube in Figure 6); the third set was scaled by direct-similarity judgment (subjective set: bottom cube in Figure 6). The circled shapes (within each set) were the shapes that underwent the three different types of scaling (unscaled, objectively scaled, and subjectively scaled). The uncircled shapes (within each set) completed the shape cubes and were generated by combining the values of curvature, tapering, and thickness borne by the scaled shapes (these extra shapes were generated to increase the task difficulty of the memory experiment but were not analyzed).

Eight four-letter nonwords ${ }^{5}$ were chosen from the ARC nonword database (Rastle, Harrington, \& Coltheart, 2001). The nonwords (baiv, fint, hong, jorl, malg, whox, verp, and yoot) were monosyllabic and had a mean bigram frequency of 5,137.625 $(S D=524.19)$.

\section{Procedure}

The experiment consisted of two stages. First, participants memorized the nonwords that would be used to label the shapes. Participants were seated in front of a computer screen and then heard all eight nonwords in random order. Participants were then instructed to verbally recall the nonwords, in any order in which they could remember them. When participants could not recall any additional nonwords, they began a new block and listened to the nonwords again. This sequence continued until participants verbally recalled all eight nonwords on two consecutive blocks, at which point the first stage was terminated.

The second stage of the experiment consisted of learning to associate the eight labels with the eight shapes within a given shape set.

Learning trials. On learning trials, each of the eight shapes was displayed for $1 \mathrm{sec}$ in the middle of the screen while its label was heard over the speakers. Once the shape disappeared, there was a 1 -sec intertrial interval, during which a blank screen was shown before the next shape was presented along with its label. This continued until all eight shapes had been presented. Shapes were presented in random order within a block of learning trials.

Test trials. On test trials, participants were presented with one of the eight shapes in the middle of the screen. Participants were instructed to "name" the shape with its associated label, and the shape remained on the screen until the experimenter recorded the participants' answers. Participants were instructed to make their best guess if they could not remember the name of the shape. Test trials continued until all eight shapes had been presented. Within a block of test trials, shapes were presented in random order. Eight learning 
trials were followed by eight test trials, with this pattern of interleaved learning and test trials continuing until 160 learning trials and 160 test trials were completed. Testing time took approximately $45 \mathrm{~min}$.

One third of the participants were presented with the eight shapes comprising the unscaled set, one third were presented with the eight shapes comprising the objective set, and the last third were presented with the eight shapes comprising the subjective set. For each group, shape-label pairings were counterbalanced across participants such that each of the eight shapes was paired with one of the eight acoustically pronounceable nonwords

\section{Results}

The results for each of the three sets were analyzed separately. For each shape set, analyses were performed only on the four shapes that were scaled, not on new shapes generated by combining the values of scaled shapes.

In terms of participants' shape-identification confusions, recall that for each set of shapes, there were four scaled shapes, and therefore six possible shape pairs that could be confused. Three pairs were one CB apart (origincurved, origin-tapered, and origin-thick), and three pairs were two CBs away (thick-tapered, thick-curved, and tapered-curved). The number of confusions for each pair of shapes was collected for all participants, and outliers were trimmed at three standard deviations. Five participants were excluded because of their poor performance- they still made confusion errors at the end of the 20 blocks. Because participants were instructed to make their best guess when unsure, all errors correspond to confusions between two shapes, for example, naming the thick shape using the origin shape's label.

For each shape set we tested two hypotheses:

Hypothesis 1. In a well-constructed psychophysical shape space, pairs of shapes whose elements are closer together will be confused more often than pairs of shapes whose elements are further apart. Therefore, shapes that were one CB apart should be confused more often than shapes that were two CBs apart. Furthermore, there should never be a violation of this CB rule. That is, there should never be an instance in which a pair of shapes lying two CBs apart is confused more often than a pair of shapes laying one $\mathrm{CB}$ apart.

Hypothesis 2. In a well-constructed psychophysical shape space, pairs of shapes that are equidistant from one another should be confused an equivalent number of times. Thus pairs of shapes that lie one CB apart should be confused equally often, and no specific pair should be confused more often than any other pair. Similarly, pairs that lie two CBs apart should be confused equally often. Separate analyses of variance were conducted for the three one-CB pairs and the three two-CB pairs. ${ }^{6}$

\section{Control Set Analyses}

Hypothesis 1. In order to see whether shapes that lie one $\mathrm{CB}$ apart were confused more often than shapes that lie two CBs apart, for each subject, confusions from the pairs of scaled shapes that lie one $\mathrm{CB}$ apart were totaled and entered as a value for one $\mathrm{CB}$ confusions. Confusions from the pairs of scaled shapes that lie two CBs apart were totaled and entered as a value for two CBs confusions. A paired $t$ test revealed that the mean number of confusions for pairs whose elements were one CB apart in psychophysical shape space (16.63 confusions) was greater than for pairs whose elements were two CBs apart (7.87 confusions) $[t(29)=6.511, p<.001]$. The mean number of confusions for each pair is displayed in Figure 7.

In a more stringent test of this hypothesis, we looked to see whether there were any violations of the CB rule using unprotected $t$ tests. The thick and the tapered shapes that lay two CBs apart (4.8 confusions) were confused significantly more often than the origin and the curved shapes that lay one CB apart (1.5 confusions) $[t(29)=3.942$, $p<.001]$, contradicting the CB rule.

Hypothesis 2. In order to see if shapes that lay one $\mathrm{CB}$ apart were confused equally often, we conducted a oneway ANOVA on the three pairs whose elements lay one $\mathrm{CB}$ apart. This one-way ANOVA revealed a main effect of pair $[F(2,58)=31.309, p<.001]$. Post hoc pairwise comparisons using unprotected $t$ tests revealed that the mean number of confusions between the origin and the tapered shapes (10.7) was significantly greater than the mean number of confusions between the origin and the thick shapes (4.4 confusions) $[t(29)=4.09, p<.001]$, which were significantly greater than the mean number of confusions between the origin and the curved shapes (1.5 confusions) $[t(29)=4.17, \mathrm{p}<.001]$.

A one-way ANOVA on the three pairs of scaled shapes that lay two CBs apart also revealed a significant main effect of pair $[F(2,56)=14.267, p<.001]$. Post hoc pairwise comparisons using unprotected $t$ tests showed that the number of confusions produced by the thick shape and the tapered shape (4.08 confusions) was significantly greater than the number of confusions generated by the thick shape and the curved shape (1.6 confusions) $[t(29)=$ $4.08, p<.001]$ and by the tapered and the curved shape (1.7 confusions) $[t(29)=3.86, p=.001]$.

\section{Objective-Set Analyses}

Hypothesis 1. A paired $t$ test revealed that the mean number of confusions for pairs whose elements lay one CB apart (1.97 confusions) was higher than for pairs whose elements lay two CBs apart ( 0.73 confusions) $[t(26)=4.703, p<.001]$, conforming to the CB rule. In a more stringent test of this hypothesis, we looked to see whether there were any violations of the $\mathrm{CB}$ rule among all possible contrasts of the six pairs of shapes. These analyses revealed two violations of the CB rule. Two pairs of shapes that lay two CBs apart - the thick and curved shapes ( $M=.73$ confusions $)$ and the thick and tapered shapes ( $M=.77$ confusions) - were each confused significantly more often than the origin and curved shape ( $M=.31$ confusions), which lay only one CB apart (both $p$ values $<.05)$.

Hypothesis 2. In order to see if shapes that lay one CB apart were confused equally often we conducted a oneway ANOVA on the three pairs of scaled shapes that lay 


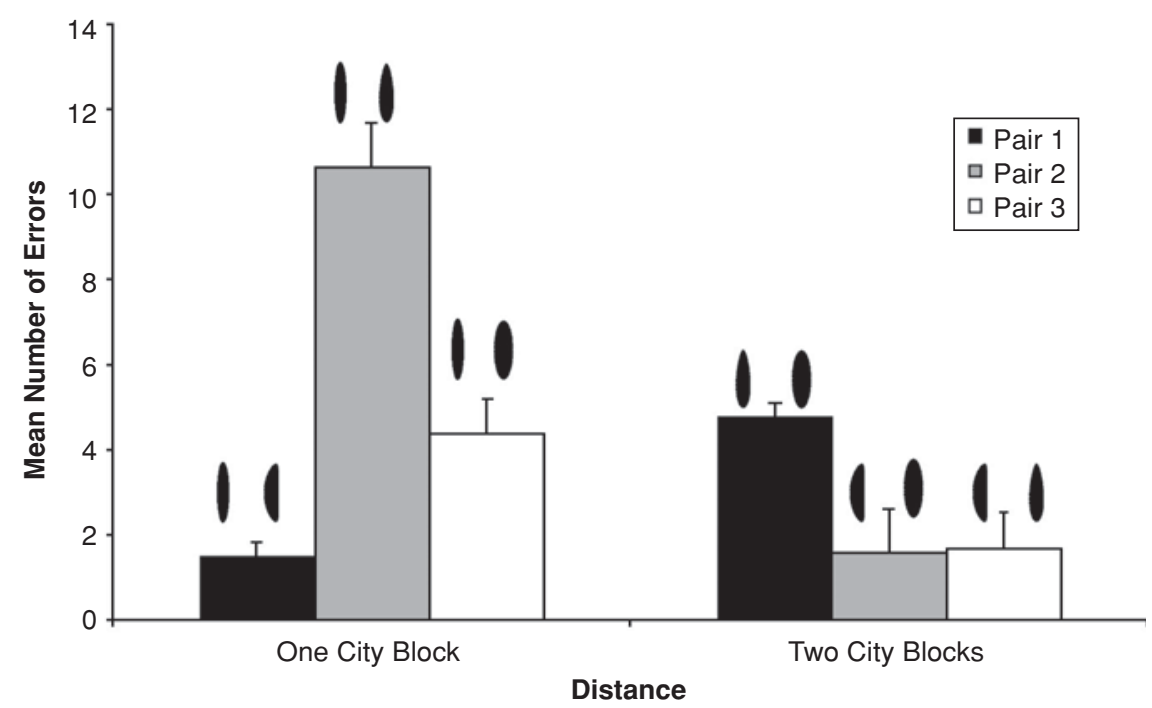

Figure 7. Mean number of errors for pairs of shapes from the control set, whose elements are one city block $(\mathrm{CB})$ apart and two $\mathrm{CBs}$ apart. The three pairs whose elements are one CB apart are origin curved (Pair 1), origin tapered (Pair 2), and origin thick (Pair 3). The three pairs whose elements are two CBs apart are tapered thick (Pair 1), curved thick (Pair 2), and curved tapered (Pair 3).

one CB apart. This one-way ANOVA revealed a significant main effect of pair $[F(2,50)=20.625, p<.001]$. The mean number of confusions for each pair is displayed in Figure 8. Post hoc pairwise comparisons revealed that the origin and thick shape ( $M=3.77$ confusions) were confused significantly more often than the origin and tapered shapes ( $M=1.85$ confusions), which in turn were confused significantly more often than the origin and curved shapes ( $M=.31$ confusions), all $p$ values $<.01$.
A second one-way ANOVA was conducted on the three pairs that were two CBs apart. Consonant with our hypothesis, the main effect of pair was not significant $[F(2,50)=.05$, n.s. $]$.

\section{Subjective-Set Analyses}

Hypothesis 1. A paired $t$ test revealed that the mean number of confusions for pairs whose elements lay one CB apart in psychophysical shape space (.89 confusions) was

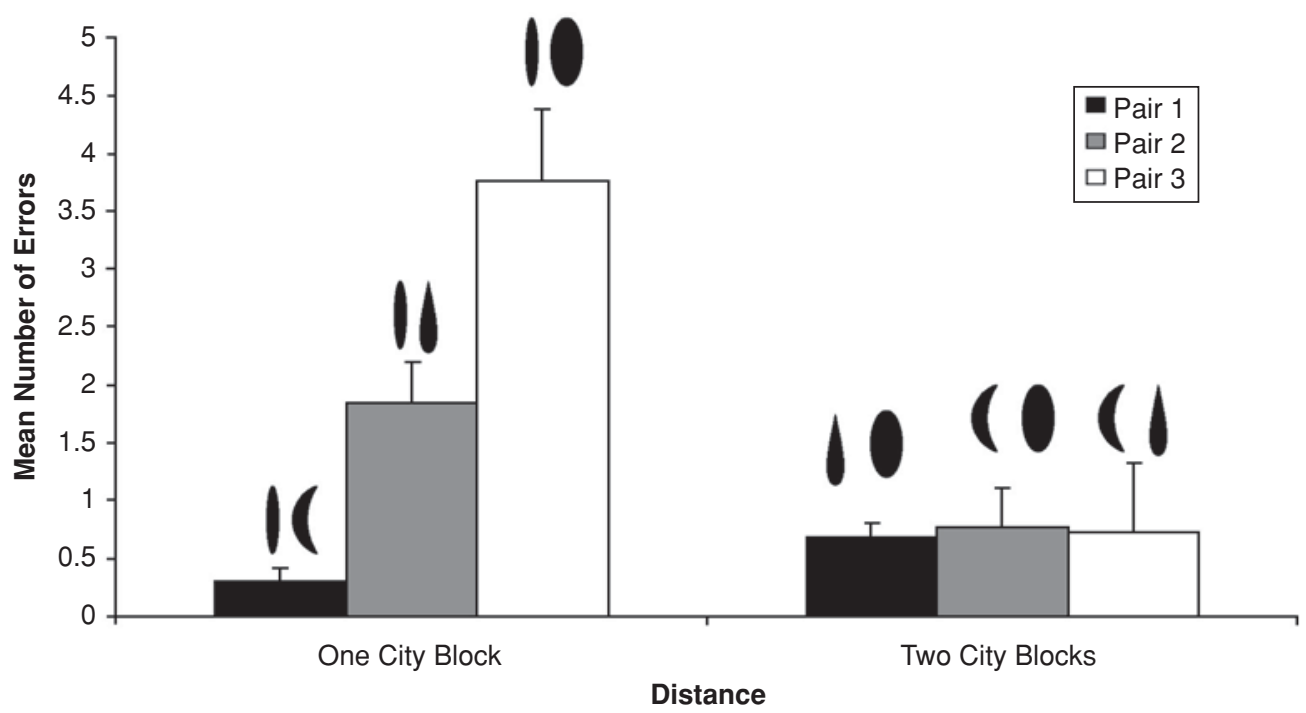

Figure 8. Mean number of errors for pairs of shapes from the objective set, whose elements are one city block (CB) apart and two CBs apart. The three pairs whose elements are one CB apart are origin curved (Pair 1), origin tapered (Pair 2), and origin thick (Pair 3). The three pairs whose elements are two CBs apart are tapered thick (Pair 1), curved thick (Pair 2), and curved tapered (Pair 3). 
greater than for pairs whose elements lay two CBs apart (.28 confusions) $[t(35)=4.814, p<.001]$. The mean number of confusions for each pair is displayed in Figure 9.

Again, in a more stringent test of this hypothesis, we looked to see whether there were any violations of the $\mathrm{CB}$ rule. For the direct set, there were no such violations. The three pairs whose shapes lie one CB apart, all produced a mean number of confusions that was greater than the mean number of confusions produced by any of the pairs whose shapes lie two CBs apart.

Hypothesis 2. A one-way ANOVA on the three pairs whose elements were one $\mathrm{CB}$ apart revealed that their mean numbers of confusions were significantly different $[F(2,70)=3.689, p<.05]$. Post hoc pairwise comparisons using unprotected $t$ tests revealed that the number of confusions produced by the origin shape and the curved shape (.53 confusions) was significantly smaller than the number of confusions generated by the origin shape and the thick shape (1.25 confusions) $[t(35)=2.476, p<$ $.05]$.

For the one-way ANOVA on the three pairs of shapes whose elements were two CBs apart, the main effect of pair was not significant $[F(2,70)=2.004$, n.s. $]$.

In summary, these results suggest that neither the shapes of the control set, the objective set, nor the subjective set perfectly reflected an ideal psychophysical shape space; however, the shape space derived using subjective scaling provided the best means of scaling shapes. For all three sets of shape spaces, pairs of shapes that lie one CB apart were confused on average more often than pairs of shapes that lie two CBs apart. However, the less formal, but more stringent test, which looked for any violations of the $\mathrm{CB}$ rule among the six key pairs of shapes within each space, revealed violations of the $\mathrm{CB}$ rule for both the unscaled shape space and the objective shape space derived using visual search. Only the shape space derived using subjective scaling conformed to the CB rule.

The three shape spaces were also evaluated by assessing whether (within each space) shape pairs that were two CBs apart yielded similar numbers of confusions. Whereas both the objective shape space and the subjective shape space met this criteria, the control shape space scaled using minimal JNDs did not. Finally, the three shape spaces were evaluated by seeing whether (within each space) shape pairs that lie one CB apart yielded similar numbers of confusions. All three shape spaces failed to meet this criterion - in all three spaces, there was a main effect of shape pair. An informal comparison of the effect sizes of this undesirable main effect calculated using $\eta^{2}$ indicated that this main effect was greatest for the control space (effect size $=.519)$, somewhat smaller for the objective space (effect size $=.45$ ), and much smaller for the subjective space (effect size $=.095$ ). This pattern of effects sizes confirms our suggestion that of the three methods, subjective scaling better estimates distance in the psychophysical shape space used by memory to identify shapes.

\section{Discussion}

Why would the subjective scaling procedure be a superior method of scaling than the objective procedure? Consider first the objective approach. It can be argued that the search procedure captures only the structural differences among the various shapes, but fails to capture other aspects of shape differences that are important in determining the psychological salience of perturbations along the three axes of curvature, tapering, and thickness. We would argue that performance on the visual search task is influenced by the same structural differences between shapes as those that are measured by algorithms

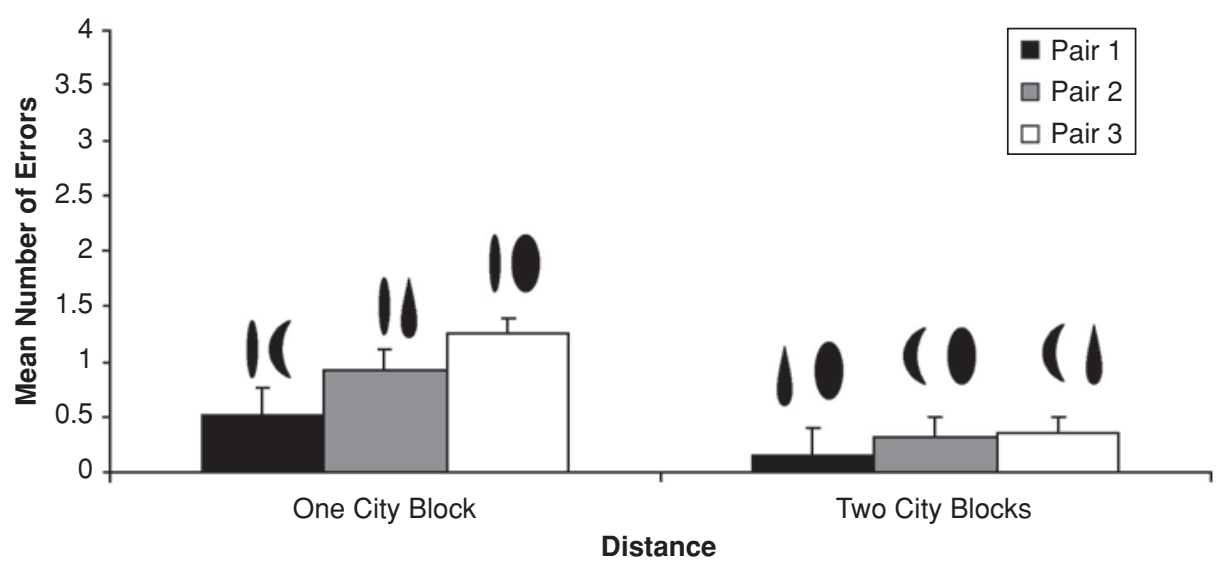

Figure 9. Mean number of errors for pairs of shapes from the subjective set, whose elements are one city block (CB) apart and two CBs apart. The three pairs whose elements are one $C B$ apart are origin curved (Pair 1), origin tapered (Pair 2), and origin thick (Pair 3). The three pairs whose elements are two CBs apart are tapered thick (Pair 1), curved thick (Pair 2), and curved tapered (Pair 3). 
that assess contour overlap. Table 3 presents the proportion of pixel overlap between the origin shape and each of the 24 shapes in the shape pool. Regression analyses indicated that pixel overlap was an excellent predictor of the obtained visual search slopes $(R=.91)$. Thus, the greater the pixel overlap between the origin shape and the distractor shapes, the steeper the slope. The finding that the objectively scaled space did a relatively poorer job of accounting for confusions in the memory task than did the subjectively scaled space indicates that the subjective procedure accessed a key factor that was missed by the objective procedure.

In the subjective scaling approach, participants may have included in their estimates of similarity important aspects of shapes that transcend purely structural characteristics. For example, if a participant is asked to rate the similarity of the origin shape and the THK1 shape, he or she may encode the differences between these shapes as only a quantitative difference - "the same shape, just fatter," for example. In this comparison, the "essence" of the shape does not change; there is only a parametric variation of the origin shape. By contrast, when asked to rate the similarity of the origin shape and the TAP 1 shape, participants may encode the differences between these shapes as a qualitative difference-"one is an ellipse, the other is tapered," or "one is symmetrical, the other is not." In this latter comparison, there is a noticeable change in the essence of the shape from the origin shape to the tapered shape (an equivalent qualitative change would also be noted from the origin shape to a curved shape). If participants take into account this factor, then relatively large perturbations of the origin shape on the dimension of thickness might be required to subjectively match perturbations of the origin shape in either curvature or tapering. Participants do indeed appear to take such factors into account. Note that the same tapered shape (TAP7) was selected in both the objective and subjective spaces. This shape can therefore be used as an anchor to contrast the thick shapes chosen in the two shape spaces. In the objective space based on visual search, the thick shape that led to an equivalent pattern of RTs as the TAP7 shape was the THK1 shape - a very small perturbation of thickness. In the subjective space, however, in order to match the TAP7 shape, the perturbation on the dimension

Table 3

Proportion of Pixel Overlap Between Scaled Shapes and the Origin Shape (Shared Proportion/Total Area)

\begin{tabular}{lccccc}
\hline $\begin{array}{l}\text { Curved } \\
\text { Shapes }\end{array}$ & $\begin{array}{c}\text { Overlap } \\
\text { in Pixels }\end{array}$ & $\begin{array}{c}\text { Tapered } \\
\text { Shapes }\end{array}$ & $\begin{array}{c}\text { Overlap } \\
\text { in Pixels }\end{array}$ & $\begin{array}{c}\text { Thick } \\
\text { Shapes }\end{array}$ & $\begin{array}{c}\text { Overlap } \\
\text { in Pixels }\end{array}$ \\
\hline CUR1 & .8808 & TAP1 & .9640 & THK1 & .7751 \\
CUR2 & .8405 & TAP2 & .9596 & THK2 & .7322 \\
CUR3 & .8181 & TAP3 & .9402 & THK3 & .6826 \\
CUR4 & .7987 & TAP4 & .9246 & THK4 & .6555 \\
CUR5 & .7787 & TAP5 & .8982 & THK5 & .6022 \\
CUR6 & .7674 & TAP6 & .8804 & THK6 & .5747 \\
CUR7 & .7427 & TAP7 & .8777 & THK7 & .5115 \\
CUR8 & .7142 & TAP8 & .8772 & THK8 & .5021 \\
\hline
\end{tabular}

Note-CUR, curved; TAP, tapered; THK, thick. of thickness had to be much larger (THK6). The relationship between the dimension of thickness and the objective and subjective scaling procedures becomes especially clear when one looks at the proportions of overlap between the origin shape and the shapes that make up the objective and subjective spaces. In the objective shape space, the proportion of pixel overlap between the origin shape with the thick shape is .7751, a value that is quite similar to the pixel overlap between the origin shape and the curved shape (.7427) and between the origin shape and the tapered shape (.8777). This makes sense, given the high correlation $(r=.91)$ between search slopes and pixel overlap - the three shapes chosen to be equal to one another on the basis of slope (and intercept) have approximately the same (high) degree of pixel overlap. In the subjective shape space, however, the proportion of pixel overlap between the origin shape and the thick shape (.5747) is much smaller than the values noted for the pixel overlap between the origin and curved shape (.7987) and between the origin and tapered shape (.8777). That is, the origin and thick shapes are more discrepant (in terms of pixel overlap) than the origin and curved or than the origin and tapered shapes, which have a similar (high) degree of pixel overlap. This finding is consistent with the idea that, because perturbations along the axis of thickness do not change the essence of the origin shape, participants require greater thickness perturbations to match perturbations of the origin shape along the axes of either curvature or tapering. ${ }^{7}$ We propose that the subjective scaling procedure's ability to capture this important contributor to shape similarity enables a better scaling of the shape space used by memory to identify shapes.

One of our objectives was to develop a set of psychophysically scaled shapes that could be used to assess shape identification. In Experiment 1, the subjectively scaled shape space was superior to the objectively scaled shape space, or the unscaled space, but still did not exactly match the shape space used to remember and identify shapes. In Experiment 2, we used the confusion rates for the various shapes employed in all the three shape spaces of Experiment 1 to create a shape space that best matched the shape space used by memory to identify shapes.

\section{EXPERIMENT 2}

In the subjective space (our best estimate of distance in psychophysical shape space so far), the number of confusions generated by the origin and the TAP7 shape $(M=$ 0.92) was not significantly different from the number of confusions produced by the origin and the THK6 shape $(M=1.25)$. Therefore, the thick shape and the tapered shapes used in the subjective set can be considered equidistant from the origin shape. The problematic pair in the subjective space was the origin and the CUR4 curved shape ( 0.53 confusions), which produced a smaller number of confusions than did the other two pairs whose elements were one $\mathrm{CB}$ apart. Thus, the perturbation of curvature in the CUR4 shape was too salient and generated too few confusions with the origin shape to match the 
TAP7 and THK6 shapes. A shape with a smaller amount of curvature than the CUR4 shape was required. The CUR1 shape was used in the control space. This shape was confused with the origin shape an average of 1.53 times. Independent sample $t$ tests revealed that confusions produced between the origin shape and CUR1 in the unscaled set ( $M=1.53$ confusions) was not significantly different from the number of confusions between the origin shape and the TAP7 shape ( $M=.92$ confusions) $[t(64)=1.73$, n.s. $]$, nor was it significantly different from the mean number of confusions between the origin shape and the THK6 ( $M=1.25$ confusions) $[t(64)=0.75$, n.s. $]$. We therefore sought to assess whether a shape space using the origin shape, CUR1, TAP7, and THK6 would reflect the psychophysical shape space used to remember and identify shapes.

\section{Method}

\section{Participants}

Participants were 27 undergraduate students registered in the Introduction to Psychology course at the University of Waterloo; each received one credit for their participation in the experiment.

\section{Materials}

A new set of eight shapes was constructed using the same method outlined in Experiment 1. For this set, CUR1 determined the value of curvature, TAP7 determined the value of tapering, and THK6 determined the value of thickness (see Figure 10).

\section{Procedure}

The procedure described in Experiment 1 was also used in Experiment 2 .

\section{Results and Discussion}

As for the previous sets, the number of confusions for each of the pairs of shapes was collected for all participants, and outliers were trimmed at three standard deviations.

Hypothesis 1. In order to see whether shapes that were one CB apart were confused more often than shapes that were two CBs apart, a paired $t$ test was conducted. The mean number of confusions for pairs whose elements lie one CB apart in psychophysical shape space (1.09 confusions) was greater than for pairs whose elements lie two CBs apart $(.24$ confusions) $[t(26)=5.969, p<.001]$. The mean number of confusions for each pair is displayed in Figure 11.

Again, we looked to see whether there were any violations of the $\mathrm{CB}$ rule. For this new set, there were no such violations. The three pairs whose shapes were one $\mathrm{CB}$ apart all produced more confusions than the confusions produced by any of the pairs whose shapes were two CBs apart.

Hypothesis 2. As in Experiment 1, we conducted a one-way ANOVA on the three pairs whose elements were one $\mathrm{CB}$ apart. Consonant with our hypothesis, the main effect of pair was not significant $[F(2,50)=0.810$, n.s.]. A one-way ANOVA on the three pairs whose elements were two CBs apart revealed that their mean numbers of confusions were also not significantly different

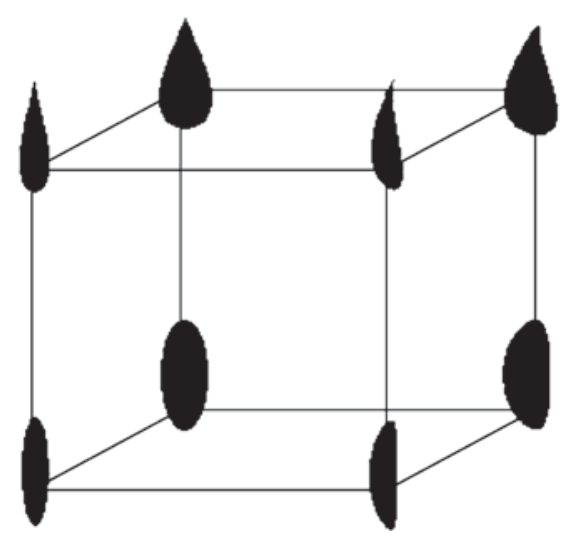

Figure 10. Set of shapes used in Experiment 2.

$[F(2,46)=0.511$, n.s.]. Effect size for this key main effect, as calculated by $\eta^{2}$, was .03 , which is smaller than the effect sizes calculated for all three previous sets of shapes. Therefore, for this set, all pairs of shapes that are one $\mathrm{CB}$ apart were confused more often than all pairs of shapes that are two CBs apart; pairs of shapes that are one $\mathrm{CB}$ apart were confused equally often, and pairs of shapes that are two CBs apart were confused equally often. The shape set constructed using the information from the three previous sets thus seems to be composed of shapes that are equidistant in the psychophysical shape space used by memory to identify shapes.

\section{GENERAL DISCUSSION}

The primary objective of this article was to identify whether objectively scaling a shape space using visual search or subjectively scaling a shape space using subjective ratings of similarity would best estimate the psychophysical shape space used to remember and identify shapes. We examined this by constructing three sets of shapes: a control set (used for comparison purposes), an objective set, and a subjective set. Each shape set was evaluated by looking at the confusion rates among the shapes comprising the shape set. A secondary objective was to develop a set of equidistant shapes that could be used in memory tasks. We therefore used the results from the three naming tasks in Experiment 1 to derive a new set of shapes that best estimated true psychophysical shape space. In this new set of shapes, all pairs of shapes that were one city block apart were confused more often than shapes that were two city blocks apart. In addition, in this new set of shapes, all shapes that were one city block from the origin shape were confused equally often, and all shapes that were two city blocks apart were confused equally often.

Both the subjectively scaled space from Experiment 1 and the new space created in Experiment 2 reveal interesting differences between the component dimensions that make up these three dimensional shape spaces. In both of these spaces, there is an equivalence in the manner in 


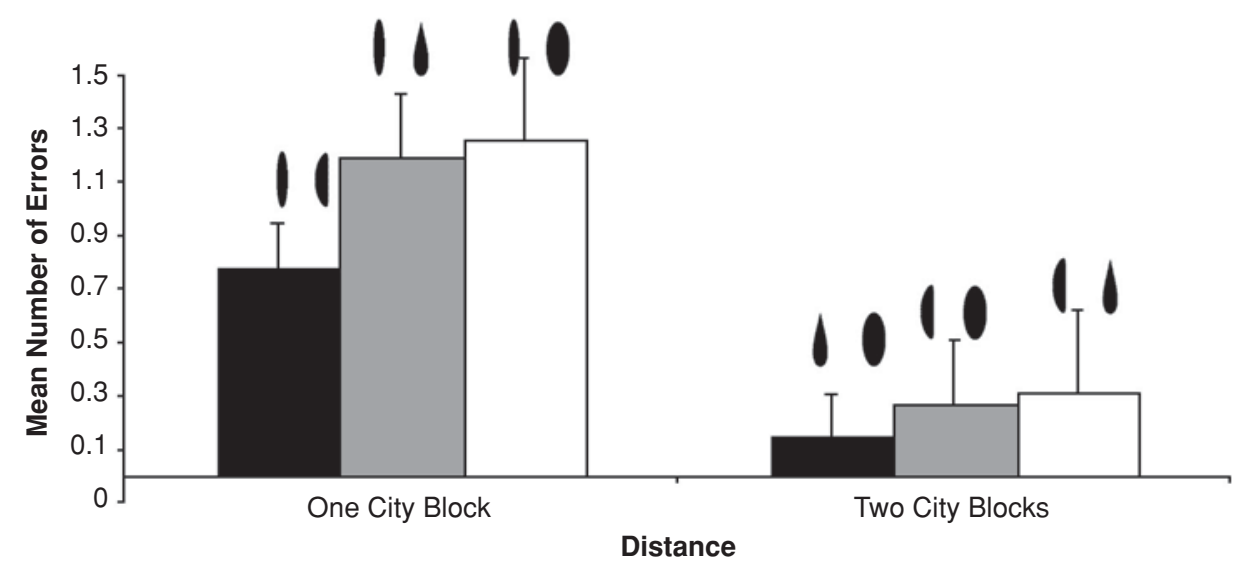

Figure 11. Mean number of errors for pairs of shapes from the new set, whose elements are one city block (CB) apart and two CBs apart. The three pairs whose elements are one CB apart are origin curved (Pair 1), origin tapered (Pair 2), and origin thick (Pair 3). The three pairs whose elements are two CBs apart are tapered thick (Pair 1), curved thick (Pair 2), and curved tapered (Pair 3).

which participants treat perturbations of the origin shape in curvature and tapering. The manner in which participants treat perturbations along the dimension of thickness appears to be different from these other two dimensions. These relations can best be understood by looking at the amount of pixel overlap between the origin shape and the shapes in the new set that provided the most adequate estimate of psychophysical shape space. In the new set, the proportion of overlap between the origin and CUR1 shape $(.8808)$ was very similar to the proportion of overlap between the origin and the TAP7 shape (.8877). However the proportion of overlap between the origin shape and the THK6 shape (.5747) was far less than the overlap noted for the other two dimensions. In other words, although the curved and tapered shapes are far more structurally similar to the origin shape than the thick shape, these four shapes generate similar numbers of errors in memory. Again these relations can be understood by looking at whether perturbations of the origin shape alter the essence of this shape. If these perturbations do alter the essence of the shape, then it appears the important variable is how similar the shapes are from one another in purely structural terms (captured by measures such as contour or pixel overlap). Perturbations along the axis of thickness, however, do not change the essence of the origin shape and subsequently, in order to generate the same degree of confusions with the origin shape as the curved and tapered exemplars, the thickness of the THK exemplar must be exaggerated (i.e., there must be far less pixel overlap between the thick shape and the origin shape than between the curved-origin or tapered-origin pairs).

The findings of the present study may provide insight into the benefits and drawbacks of how researchers have sought to account for identification deficits among patients with temporal lobe damage using line drawings. The current findings highlight how objective measures such as pixel or contour overlap will capture certain aspects of how shapes are stored in memory, but miss out on other aspects (e.g., fail to capture the high salience of changes to the essence of a shape vs. two shapes that have the same essence). The current findings also suggest that subjective means of assessing shape similarity may be superior to purely objective means of assessing similarity because they do capture the factors missed by these objective methods.

Interestingly, even though the subjective approach was superior to the objective approach in scaling thickness, the subjective approach seemed to underestimate the salience of perturbations of curvature in a psychophysical shape space used to remember and identify shapes. Using subjective scaling, the CUR4 was judged to be equidistant from the origin shape when compared to TAP7 and THK6, yet the CUR4 produced fewer confusions with the origin shape than either TAP7 or THK6.

Why might curvature be more salient in memory than in online perceptual judgments? Characteristics inherent to the visual dimensions of the shapes, like the configuration of the axes of symmetry and elongation and where the bulk of the shape is distributed, may make perturbations along the axis of curvature more salient than perturbations along the axis of tapering or thickness (Hamada \& Ishihara, 1988; Quinlan \& Humphreys, 1993; Sekuler \& Swimmer, 2000). For the curved shape, the axis of elongation is vertical and the axis of symmetry is horizontal. In contrast, the axes of elongation and of symmetry were aligned in the origin, the tapered, and the thick shapes. In addition the bulk of the curved shape fell away from the horizontal axis of symmetry, whereas the bulk of the origin, the tapered, and the thick shapes all followed the vertical axis of symmetry. Hamada and Ishihara (1988) suggested that this would give a curved shape a spatial organization that differed holistically from the spatial organization of uncurved shapes like the origin, the tapered, and the thick shapes we used in our experiments. These factors all suggest the curved shape may "stand out" from the origin, tapered, and thick shapes. Why would these factors be more relevant to the memory task than to the subjective similarity task? Note that in the subjective simi- 
larity judgment task, when curved shapes are the targets, they are contrasted with two of the key shapes: the origin shape and the tapered shape. In the memory task, however, participants are forced to consider (and contrast) the curved shape against all three of the key shapes (the origin shape, the tapered shape, and the thick shape). This extra contrast with the thick shape may serve to increase the saliency of factors that are unique to the curved shape (e.g., its asymmetry with respect to the vertical axis and the fact that only for this exemplar is the bulk of the shape falling away from this vertical axis). Note that the shape set that ultimately provided the best scaling of shapes in memory drew upon a combination of techniques. Arguably, objective measures such as pixel overlap appear to be excellent means of accounting for memory confusions among shapes with different essences (e.g., the origin versus the tapered shape, the origin versus the curved shapes), but subjective measures are required to scale the distance between different shapes that have the same shape essence (e.g., the origin and the thick shape). By combining these objective and subjective methods, we were able to construct a set of shapes whose distance in shape space accounted for memory confusions.

The key characteristic of this set of shapes is that there is an equal amount of visual similarity on each of the three dimensions used-curvature, tapering, and thickness. This is why we observed an equal number of confusions for each pair whose elements were at a given distance in psychophysical shape space. Because we used nonword labels, we can also assume that errors are due only to the visual similarity between the shapes.

This scaled shape space can be used as a basic tool in understanding the object identification errors made by patients with category-specific deficits. If brain damage has preferentially caused problems for patients processing perturbations along certain shape dimensions (e.g., curvature) but not along others (thickness and tapering), then predictable patterns of deficits would ensue (e.g., the patient would confuse the origin and curved shape more often than the origin and thick or the origin and tapered shapes). Because these shapes have been scaled to reflect healthy individuals' memory confusions, any perturbations of this space can pick up these specific types of memory deficit. Importantly, this scaled shape space may even be able to provide a window on semantic similarity.

Previously, we alluded to an experiment with the CSVA Patient E.L.M. who attempted to "name" shapes. Unlike in the current experiments, the labels used to name shapes were those of stringed musical instruments. In the experiment with E.L.M., different numbers of confusions were noted between shapes that were one CB apart. With these unscaled shapes, we did not know whether these differences were due to similarity in psychophysical shape space OR because of semantic similarity between the labels attached to the shapes. With the scaled set of shapes that we have derived in Experiment 2, however, one could effectively measure semantic similarity and its effects on object identification. If one wished to know whether a gui- tar was closer in semantic space to a banjo or a violin, one might pair the guitar with the origin shape, the banjo to the THK6 shape, and the violin to the TAP7 shape, and use this naming paradigm to find the answer. Because these three shapes are equidistant in shape space, any differences in confusability must be due to semantic distance.

\section{CONCLUSION}

The first goal of this article was to identify which approach best estimated distance reflecting a veridical psychophysical shape space. A set of eight shapes was constructed using each of three approaches, and participants were asked to identify these eight shapes in a naming task. How well JND-like scaling, objective scaling using visual search, or subjective scaling using similarity estimates reflected true psychophysical shape space was assessed by looking at the memory confusions among the shapes. The results suggest that although neither of the approaches perfectly reflected a true psychophysical shape space, subjective scaling was a better estimator of distance in psychophysical shape space than objective scaling was.

A second goal of this article was to develop a set of shapes that could be used to investigate object identification performance in patients as well as neurologically intact participants. The number of confusions observed in the three sets constructed was used to generate a new set of shapes that was a better estimator of distance in true psychophysical shape space. A naming task verified that the shapes of this new set were equidistant in psychophysical shape space. This shape space can be used as a research tool for investigating the factors that influence object identification in patients and healthy observers.

\section{REFERENCES}

Ashby, F. G., \& Lee, W. W. (1991). Predicting similarity and categorization from identification. Journal of Experimental Psychology: General, 120, 150-172.

Biederman, I. (1987). Recognition-by-components: A theory of human image understanding. Psychological Review, 94, 115-147.

Brown, J. M., Weisstein, N., \& MaY, J. G. (1992). Visual search for simple volumetric shapes. Perception \& Psychophysics, 51, 40-48.

Cavanagh, P., Arguin, M., \& Treisman, A. (1990). Effect of surface medium on visual search for orientation and size features. Journal of Experimental Psychology: Human Perception \& Performance, 16, 479-491.

Dixon, M. J., Bub, D. N., \& Arguin, M. (1997). The interaction of object form and object meaning in the identification performance of a patient with category-specific visual agnosia. Cognitive Neuropsychology, 14, 1085-1130.

Dixon, M. J., Bub, D. N., ChertKow, H., \& Arguin, M. (1999). Object identification deficits in dementia of the Alzheimer type: Combined effects of semantic and visual proximity. Journal of the International Neuropsychological Society, 5, 330-345.

Dixon, M. J., Bub, D., Lupien, S., Pavate, S., Malette, J., \& Nair, N. P. V. (1998). Selective attention for shapes in patients with Alzheimer's disease [Proceedings of the 26th annual meeting of the International Neuropsychological Society]. Journal of the International Neuropsychological Society, 4, 55.

Dixon, M. J., Desmarais, G., Gojmerac, C., Schweizer, T. A., \& Bub, D. N. (2002). The role of premorbid expertise on object identification in category-specific visual agnosia. Cognitive Neuropsychology, 19, 401-419. 
Dudek, G., Arguin, M., Dixon, M., \& Bub, D. (1997). Coding simple shapes for recognition and the human integration of shape descriptors. In M. Jenkin \& L. Harris (Eds.), Computational and psychophysical mechanisms of visual coding (pp. 44-60). Cambridge: Cambridge University Press.

Forde, E. M. E., Francis, D., Riddoch, M. J., Rumiati, R. I., \& HumPHREYS, G. W. (1997). On the links between visual knowledge and naming: A single case study of a patient with a category-specific impairment for living things. Cognitive Neuropsychology, 14, 403-458.

Hamada, J., \& Ishinara, T. (1988). Complexity and goodness of dot patterns varying in symmetry. Psychological Research, 50, 155-161.

HeLm, C. E. (1964). Multidimensional ratio scaling analysis of perceived color relations. Journal of the Optical Society of America, 54, 256-262.

Lockhead, G. R., \& KING, M. C. (1977). Classifying integral stimuli. Journal of Experimental Psychology: Human Perception \& Performance, 3, 436-443.

Podgorny, P., \& Garner, W. R. (1979). Reaction time as a measure of inter- and intraobject visual similarity: Letters of the alphabet. Perception \& Psychophysics, 26, 37-52.

Quinlan, P. T., \& Humphreys, G. W. (1993). Perceptual frames of reference and two-dimensional shape recognition: Further examination of internal axes. Perception, 22, 1343-1364.

Rastle, K., Harrington, J., \& Coltheart M. (2001, February). ARC psycholinguistic database [Online]. Available at http//www.maccs. mq.edu.au/ nwdb/

Sekuler, A. B., \& Swimmer, M. B. (2000). Interactions between symmetry and elongation in determining reference frames for object perception. Canadian Journal of Experimental Psychology, 54, 42-56.

SHEPARD, R. N. (1987). Toward a universal law of generalization for psychological science. Science, 237, 1317-1323.

STERNBERG, S. (1969a). The discovery of processing stages: Extensions of Donders' method. In W. G. Koster (Ed.), Attention and performance II. Amsterdam: North-Holland.

SternBerg, S. (1969b). Memory-scanning: Mental processes revealed by reaction-time experiments. American Scientist, 57, 421-457.

Tomonaga, M., \& Matsuzawa, T. (1992). Perception of complex geometric figures in chimpanzees (Pan troglodytes) and humans (Homo sapiens): Analyses of visual similarity on the basis of choice reaction time. Journal of Comparative Psychology, 106, 43-52.

Tranel, D., Logan, C. G., Frank, R. J., \& Damasio, A. R. (1997). Explaining category-related effects in the retrieval of conceptual and lexical knowledge for concrete entities: Operationalization and analysis of factors. Neuropsychologia, 35, 1329-1339.

Treisman, A., \& Gormican, S. (1988). Feature analysis in early vision: Evidence from search asymmetries. Psychological Review, 95, 1548.

Treisman, A., \& Souther, J. (1985). Search asymmetry: A diagnostic for preattentive processing of separable features. Journal of Experimental Psychology: General, 114, 285-310.

\section{NOTES}

1. One may ask why novel shapes should be used rather than other geometrical shapes such as rectangles and triangles. The main advantage of novel shapes is that they have less semantic baggage than other geometrical shapes. Geometrical shapes such as rectangles, squares, and triangles already have learned associations to identities or labels that may influence the similarity between them. That is, two structurally dissimilar triangles may be regarded as similar because they bear the same name. Novel shapes do not bear labels, and hence similarity may be more purely based on their visual properties (shape) rather than nonvisual properties (e.g., labels).

2. Research on visual search suggests that dramatically different search slopes can emerge depending on whether a particular shape serves as a target or a distractor. Using the same straight and curved lines in different displays, Treisman and Gormican (1988) showed relatively steep search slopes when searching for a straight line amid curved lines, but flat slopes when searching for a curved line amid straight lines. Brown, Weisstein, and May (1992) have observed similar patterns using straight and curved volumetric shapes. Treisman and Souther (1985) have also shown that searching for targets that have a distinct feature (e.g., curvature) is far more efficient than searching for targets that are defined by the absence of these features (lack of curvature). To avoid floor effects associated with flat search slopes, we therefore chose to use the origin shape as the target and to vary distractors in the displays. By using this thin, uncurved, and untapered shape as the target search, slopes had the potential to become either shallower or steeper depending on the type of distractors that we employed.

3. Intercepts in a search task may be indicative of processes other than search efficiency, which is influenced by visual similarity (Cavanagh, Arguin, \& Treisman, 1990; Sternberg, 1969a, 1969b). However, the aim here is to develop a set of three shapes that have an equal amount of similarity to an origin shape. Although intercepts may be indicative of factors other than visual similarity, including this measure as an indicator of similarity should create a more homogeneous set of shapes. Also, the triad of shapes chosen using both slopes and intercepts (CUR7, TAP7, THK1) and the triad identified using only the most similar slopes (CUR5, TAP7, THK1) are minimally different.

4. Recall that our goal was to compare sets of shapes scaled using visual search to sets of shapes scaled using a more direct scaling procedure. For the search set, we derived three shapes - one curved, one tapered, and one thick - that were hypothetically psychophysically equidistant from an "origin shape" that was neither curved, tapered, nor thick. In order to meaningfully compare the set of shapes derived using RT to a set of shapes derived using direct scaling, we used the origin shape and the tapered shape from the search set as anchor shapes.

5. In order to get at an unbiased estimate of psychophysical shape space, we needed to use labels without semantic referents. If we were to use words such as carrot, cucumber, and balloon, for example, the semantics attached to these words could warp the psychophysical shape space of the shapes to which they were attached. "Balloon" could make the shape attached to it easier to remember because it is not a vegetable and therefore "stands out" with respect to the other two triad members. Therefore, semantics can "add distance" between shapes. Dixon et al. (1997) demonstrated that the same set of shapes was confused more often if the labels attached to them were similar in meaning, and they were confused less often if the labels attached to them were different in meaning. To avoid the problem of semantics altogether, in Experiment 2 we used nonwords as labels because they have no meaning attached to them and thus have no semantics that could warp psychophysical shape space.

6. The three pairs whose elements were one CB apart (origin-curved, origin-tapered, and origin-thick) did not correspond to the three pairs whose elements were two CBs apart (curved-tapered, curved-thick, and tapered-thick), and there was no a priori or logical reason to associate them. Thus the three pairs whose elements were one CB apart were analyzed independently of the three pairs whose elements were two CBs apart.

7. We note that in the recognition-by-components theory of object identification Biederman (1987), there are curved geons and tapered geons. The dimension of thickness served only to parametrically vary the appearance of these geons. In other words, although one curved geon may have been thicker than another, it did not change the essence of the shape (it was still the same geon).

(Manuscript submitted March 25, 2003; revision accepted for publication November 19, 2004.) 